
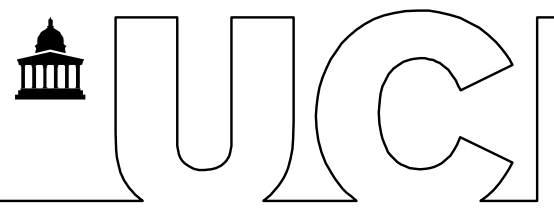

Tillmann, J; Olguin, A; Tuomainen, J; Swettenham, J; (2015) The Effect of Visual Perceptual Load on Auditory Awareness in Autism Spectrum Disorder. Journal of Autism and Developmental Disorders, 45 (10) pp. 3297-3307. 10.1007/s10803-015-2491-9. Downloaded from UCL Discovery:

http://discovery.ucl.ac.uk/1469761

\title{
ARTICLE
}

\section{The Effect of Visual Perceptual Load on Auditory Awareness in Autism Spectrum Disorder}

\author{
Tillman, Julian ${ }^{1}$; Olguin, Andrea ${ }^{1}$; Tuomainen, Jyrki; Swettenham, John ${ }^{1}$ \\ ${ }^{1}$ Department of Developmental Science, University College London \\ ${ }^{2}$ Speech, Hearing and Phonetic Sciences, University College London
}

\begin{abstract}
Recent work on visual selective attention has shown that individuals with Autism Spectrum Disorder (ASD) demonstrate an increased perceptual capacity. The current study examined whether increasing visual perceptual load also has less of an effect on auditory awareness in children with ASD. Participants performed either a high- or low load version of a line discrimination task. On a critical trial, an unexpected, task-irrelevant auditory stimulus was played concurrently with the visual stimulus. In contrast to typically developing (TD) children, children with ASD demonstrated similar detection rates across perceptual load conditions, and reported greater awareness than TD children in the high perceptual load condition. These findings suggest an increased perceptual capacity in children with ASD that operates across sensory modalities.
\end{abstract}

Keywords: Autism Spectrum Disorder; auditory awareness; attention; perceptual load

Autism Spectrum Disorder (ASD) is a life-long condition, characterised by impairments in social communication and the presence of restricted and fixated interests (DSM-V; American Psychiatric Association, 2013). Alongside these core features, individuals with ASD also display an unusual profile of perceptual strengths and weaknesses both in the visual and auditory domain (Simmons et al. 2009; O'Connor, 2012). Since perception provides an important input channel for higher-order social and cognitive processes (Johnson, 2005), understanding how individuals with ASD perceive their environment may be an important step to understanding the social and cognitive deficits associated with the disorder.

The most prominent theories attempting to account for perceptual anomalies in ASD, such as Weak Central Coherence (Frith, 1989; Happé and Frith, 2006) and Enhanced Perceptual Functioning (Mottron et al. 2006), have focussed on explaining enhanced processing of local, low-level information or scene-detail in stimuli. For example, in the visual domain, a preference for local processing could be reflected by performance on the Navon task (Plaisted et al. 1999), the embedded figures task (Shah and Frith, 1999) and the block design task (Shah and Frith, 1993; Happé, 1996); and in the auditory domain by a higher incidence of perfect pitch (e.g. Heaton, 2003) and enhanced frequency discrimination skills, at least in a subgroup of those with ASD (Bonnel et al. 2010; Jones et al. 2009).

Recently, there has been a new proposal that ASD might be characterised by an increased perceptual capacity (Remington et al. 2009, Remington et al. 2012b, Swettenham et al. 
2014). This proposal attempts to account for enhanced processing of low-level information and scene-detail, as well discrepant findings in visual selective attention in ASD, where some studies suggest high distractibility (e.g. Adams and Jarrold, 2012; Burack, 1994; Christ et al. 2007; Geurts et al. 2008) and others suggest greater focus (e.g. O'Riordan, 2004; O'Riordan et al. 2001; Plaisted et al. 1998). This work has been based on the idea of applying Lavie's Load theory of selective attention in ASD (Lavie, 1995).

According to Load theory, the extent to which irrelevant distractors will be processed depends on the level of perceptual load of the task. The perceptual load of a task can be manipulated by increasing the number of different task-relevant items (e.g. by increasing the number of additional visual stimuli in a search display) or by increasing the perceptual processing requirements (e.g. making a line discrimination task more subtle) (Lavie, 1995; Lavie and Tsal, 1994). It is not just the number of units in a task-relevant display which constitutes perceptual load, but even for single item displays "perceptual load correlates with the amount of information required to process each unit in order to produce the required perceptual response" (Lavie and Tsal, 1994, p. 185). A key assumption of the model is that while perceptual processing is limited in capacity, it proceeds automatically and involuntarily until it runs out of capacity (Lavie, 2005; Lavie, 2010). It follows then that under conditions of low perceptual load any spare capacity after the processing of task-relevant stimuli automatically "spills-over" to process irrelevant distractors. In tasks of high perceptual load however, full processing capacity is engaged, leaving no additional capacity to process irrelevant distractors (Lavie, 1995). It follows then that the level of perceptual load at which task-irrelevant stimuli are no longer processed relates to an individual's perceptual capacity. It is important to note at this point that the effects of perceptual load on attention can be distinguished from the effects of task difficulty. Perceptual load can be manipulated by increasing the number of task-relevant items or by increasing perceptual processing requirements, thereby placing higher attentional demands on available resources (i.e. increasing data "resource limits" (Lavie, 2005; Lavie, 2010). Task difficulty on the other hand can be increased by manipulations of extreme sensory degradation, altering sensory "data limits" (e.g. by manipulating contrast or size of a target) (Lavie and de Fockert, 2003). Lavie and de Fockert (2003) demonstrated that whereas an increase in perceptual load resulted in reduced distractor processing, task difficulty (as defined above) disrupts task performance while having no effect on distractor processing.

The main competing account to Load theory is Dilution theory (Tsal and Benoni, 2010a, $2010 \mathrm{~b})$. On this account, the reduction in distractor interference observed under high perceptual load (in tasks manipulating search set size) occurs as a result of the diluting effect of the distractor by neutral items. To demonstrate this, Tsal and Benoni (2010a) presented participants with a low perceptual load/high dilution search task, where the number of non-target items matched a typical high perceptual load display, but with these non-target items being clearly distinguishable from the target item (i.e. red target among black non-targets). In this condition, ostensibly a low load condition as the target "pops-out", the mere presence of non-target items (neutral letters) was sufficient to eliminate distractor processing. According to Tsal and Benoni (2010a, 2010b), the features of the non-target search items (e.g. neutral letters) competed with those of the distractor, thus degrading the quality of its visual representation and leading to reduced distractor processing. Lavie however has attempted to counter this claim by demonstrating that in low perceptual load/high search set size displays, capacity in fact spills over into processing the non-target items in the search array, therefore ruling out an alternative explanation in terms of dilution (Lavie and Torralbo, 2010). Lavie and Torralbo (2010) also point out that their interpretation of perceptual load effects in terms of dilution only pertain to one specific manipulation of perceptual load. That is, manipulating the relevant search set size and measuring its effect on distractor response competition effects. However, recall that apart from increasing the number of relevant items in a search task (e.g. by increasing the search set size), perceptual load can be manipulated by increasing the processing requirements of items in the relevant 
task (Lavie, 1995; 2005). For example, a more subtle line discrimination task should demand considerably more attentional resources than a gross line discrimination task. There have been a number of published studies that have manipulated the perceptual load of a singleelement display (e.g. line discrimination) and demonstrated reduced distractor interference with increased perceptual load either behaviourally (Cartwright-Finch and Lavie, 2007; Remington et al. 2014; Macdonald and Lavie, 2011; Swettenham et al. 2014) or by reduced neural responses evoked by task-irrelevant information in high perceptual load single element displays (Schwartz et al. 2005; Carmel et al. 2011).

Remington et al. (2009), using a hybrid between a visual search task (Treisman and Gelade, 1980) and a flanker task (Erikson and Erikson, 1974), assessed the extent to which visual distractors were processed under different levels of visual perceptual load in a group of adults with ASD and neurotypical adults (matched for chronological age and IQ). When the perceptual load of the task was low (1 or 2 items in the search array), both groups were slower to search for a target in the presence of incompatible distractors compared to neutral distractors. This interference effect suggests that both participant groups were processing distractors, despite being told to ignore them. However, when perceptual load was higher (4 items in the search array) only the ASD group displayed an interference effect, suggesting that they continued to process distractors at a higher level of perceptual load than the comparison group. At even higher levels of perceptual load (6 search array items) there was no interference effect in either group. These findings strongly suggest that individuals with ASD have a higher perceptual capacity. Capacity was not exhausted until there were 6 items in the search array for the ASD group, compared to 4 items in the search array for the neurotypical group.

Two recent studies which directly manipulated perceptual load as a dependent variable have provided further support for the claim that ASD is characterised by an increased perceptual capacity. Remington, Swettenham and Lavie (2012b) demonstrated higher levels of distractor detection at high levels of perceptual load in adults with ASD compared to a control group. In addition to a central letter search task participants were asked to report the presence or absence of a shape which had appeared at the same time as the central letter display on $50 \%$ of trials. For control participants, detection sensitivity for the shape declined as the visual perceptual load of the central letter search task increased. In contrast, detection sensitivity remained high even under conditions of high visual perceptual load in participants with ASD, despite no cost in accuracy or reaction time on the central search task. Swettenham et al. (2014) also asked participants to report awareness of a taskirrelevant stimulus, however this time the appearance of the irrelevant stimulus was completely unexpected. Using an inattentional blindness paradigm, Swettenham et al. (2014) presented children with ASD and age-and IQ-matched TD children with either a subtle- (high perceptual load) or gross line discrimination task (low perceptual load). On a critical trial, an unexpected, additional visual stimulus (black square shape) was presented together with the central line discrimination cross in one of four possible locations in the periphery. Once participants had responded to the line discrimination they were asked whether they had noticed anything else. The results indicated that while high visual perceptual load reduced awareness of an unexpected visual stimulus in typically developing (TD) children, awareness rates remained high for children with ASD even when the perceptual load of the central task was high. As the effects of perceptual load on attention in ASD have to date only been shown within the visual modality, it is currently not known whether this extends to contexts of cross-modal selective attention. Indeed, the only studies that have investigated cross-modal attention in ASD have produced conflicting results and did not manipulate the perceptual load of the task. Casey et al. (1993) for instance showed that autistic savants were less accurate (i.e. made more omission errors) than age-, and gender-matched controls at detecting visual and auditory target stimuli presented simultaneously in a continuous performance test (CPT). This suggested to the authors that autistic savants are impaired in their ability to respond to more than one task simultaneously. 
However, the small sample size in each group $(n=10)$, substantially lower IQ scores in the ASD group, and ceiling performance of TD participants on both unisensory visual/auditory and cross-modal CPT tasks suggest the need for further exploration.

In contrast, Ciesielski et al. (1995) found similar behavioural performance between groups on an auditory-visual divided attention task, yet an atypical activation pattern of event-related potentials (ERPs) as measured by the slow negative wave (SNW) in adolescents with highfunctioning ASD relative to controls. The slow negative wave is thought to reflect voluntary attention towards target stimuli and is modulated by increasing task demands. This effect was observed in TD subjects, with reduced frontal auditory and visual SNW's in the divided attention condition compared to the focused attention condition. Such task related modulation of the SNW however was not found in participants with ASD, suggestive of atypical voluntary attentive processing at the neural level. Nonetheless, individuals with ASD were able to perform the task as well as TD controls. This dissociation suggests that individuals with ASD did not need to deploy attention to the same extent as TD controls to be able to perform the task. It is difficult to tell whether these findings could be explained by an increased perceptual capacity in ASD. For example, in the divided attention condition, stimuli were presented in a serial order ( 2 flashes and 2 sounds presented in a random sequence), which suggests that performance on this task was dependent on rapid switching between target items rather than being able to process more perceptual information simultaneously. Perceptual load was also not directly manipulated in either of the above studies and hence it remains unclear whether the effects of perceptual load in ASD in the visual domain also translate to cross-modal contexts of attention.

In the current study, we examined whether increasing perceptual load in the visual modality influences awareness in the auditory modality in a group of children with ASD and a neurotypical comparison group. Does an increased perceptual capacity in ASD influence perceptual awareness across modalities?

In neurotypical adults, it has now been shown that increasing the perceptual load in a task reduces the incidence of inattentional deafness (Macdonald and Lavie, 2011; Raveh and Lavie, 2015). Inattentional deafness refers to the phenomenon that participants often fail to notice an unexpected auditory stimulus on a critical trial when their attention is engaged in the primary task, yet report awareness on a following control trial when they are told to ignore the primary task stimulus and instead pay attention to anything else. Across three experiments, Macdonald and Lavie (2011) demonstrated that whilst awareness rates were not affected on a gross line discrimination task (low perceptual load), significantly fewer participants reported awareness during a subtle line discrimination (high perceptual load). Importantly, these effects remained when high- and low perceptual load trials were intermixed, therefore ruling out other explanations in terms of top-down factors such as load anticipation (cf. Handy and Mangun, 2000; Murray and Jones, 2002; Theeuwes et al. 2004). The authors concluded from these findings that vision and hearing share a common attentional resource, as performing a visual task with high perceptual load exhausted all available attentional capacity and led to reduced awareness of an additional auditory stimulus. In the low perceptual load task however, attentional resources were not fully depleted and available resources automatically spilled-over into processing of the auditory stimulus.

Conflicting evidence was recently provided by a study investigating the effect of visual perceptual load on auditory distractor processing across typical development. Matusz et al. (2015) asked 6-year-olds, 11-year-olds and adults to search for a coloured shape according to a conjunction of features (a red square or a green circle) amongst other non-target shapes (red and green triangles, circles, and squares). The perceptual load of the task was manipulated by either presenting the target shape alone (low perceptual load), or together with three non-target shapes (high perceptual load) in one of six possible locations. Auditory 
distractors (voice recordings of a person saying "red" or "green") were presented through speakers and were either compatible or incompatible with the target response. The results indicated that auditory distractors were processed by all age groups regardless of the level of perceptual load. This would suggest that across development, processing of auditory distractors is not dependent on the level of visual perceptual load. However, in this study, the auditory distractor was presented for a longer duration $(500 \mathrm{~ms})$ than the visual search display $(200 \mathrm{~ms})$, suggesting that the longer temporal overlap between visual and auditory stimuli in the high load condition (due to longer search times) could have offset any perceptual load effect. In addition, this experimental design only provides an indirect measure of distractor perception (via RT performance). Indeed, reaction time costs incurred on incompatible versus compatible trials may not necessarily reflect increased distractor perception, but instead relate to post-perceptual processes such as response selection. Based on the findings from neurotypical adults that attentional resources are allocated across sensory modalities (Macdonald and Lavie, 2011; Raveh and Lavie, 2015) and the recent findings suggesting that perceptual capacity is increased in ASD (Remington et al. 2009, 2012b; Swettenham et al. 2014), we reasoned that children with ASD would also show reduced inattentional deafness under high visual perceptual load.

\section{Methods}

Participants

44 typically developing (TD) children, attending a local mainstream school, and 29 children with a diagnosis of Autism Spectrum Disorder (ASD), attending a school specifically for children with a diagnosis of ASD, took part in the study. Informed consent was received prior to the experiment. All participants with ASD had received a clinical diagnosis of ASD from a trained, independent clinician using the ADOS and following criteria listed in the Diagnostic and Statistical Manual of Mental Disorders, Fourth Edition (American Psychiatric Association, 1994). ASD diagnosis was also confirmed at the time of testing using the Social Communication Questionnaire (Rutter et al. 2003); with all participants in the ASD group scoring above the recommended cut-off score of 15 . None of the participants had any other neurological disorder. The experimental design of this study is a factorial design with two independent predictors ('Perceptual load': high vs. low and 'Group': ASD vs. TD), with all participants being randomly distributed across the two 'perceptual load' conditions. Participants were excluded if they obtained a score of less than five correct on the six noncritical trials, were incorrect on the critical trial or were unable to hear the tone on the control trial. These exclusion criteria were necessary to make sure that all participants were engaging with the primary task on the critical trial that featured the additional auditory stimulus. Three children with ASD scored less than five correct non-critical trials (all in the high perceptual load condition) and therefore were removed from the sample. Including these participants in a separate analysis produced the same findings reported later. The remaining 26 children with ASD (14 in the low load condition and 12 in the high load condition) and 44 typically developing children (21 in the low load condition and 23 in the high load condition) were matched for chronological age and non-verbal ability scores using the Raven's Standard Progressive Matrices (Raven, Raven and Court, 1998) (see Table 1). An independent samples t-test indicated that there were no significant differences in nonverbal ability scores and chronological age between groups, between perceptual load conditions and between group/perceptual load conditions (maximum t-value $=1.84$, minimum $p$-value $=0.07$ ). 
Table 1 Descriptive statistics for each group

\begin{tabular}{|c|c|c|c|c|c|}
\hline Group & Statistic & $\begin{array}{lr}\mathrm{CA} & \text { range } \\
\text { (years : months) }\end{array}$ & $\begin{array}{l}\text { Raven's } \\
\text { Score }\end{array}$ & $\begin{array}{l}\text { Cross- } \\
\text { judgment task }\end{array}$ & $\begin{array}{l}\text { SCQ } \\
\text { score }\end{array}$ \\
\hline \multicolumn{6}{|l|}{$\operatorname{ASD}(n=26)$} \\
\hline Low load $(n=14)$ & $\begin{array}{l}\text { M } \\
\text { SD } \\
\text { Range }\end{array}$ & $\begin{array}{l}10: 4 \\
0: 6 \\
9: 3-11: 5\end{array}$ & $\begin{array}{l}34.5 \\
6.9 \\
24-45\end{array}$ & $\begin{array}{l}6.64 \\
0.63\end{array}$ & $\begin{array}{l}19.71 \\
3.79 \\
15-24\end{array}$ \\
\hline High load $(n=12)$ & $\begin{array}{l}\text { M } \\
\text { SD } \\
\text { Range }\end{array}$ & $\begin{array}{l}10: 6 \\
0: 6 \\
9: 6-11: 9\end{array}$ & $\begin{array}{l}36.7 \\
5.1 \\
24-46\end{array}$ & $\begin{array}{l}6.58 \\
0.52\end{array}$ & $\begin{array}{l}18.92 \\
2.84 \\
15-26\end{array}$ \\
\hline \multicolumn{6}{|l|}{ TD $(n=44)$} \\
\hline Low load $(n=21)$ & $\begin{array}{l}\text { M } \\
\text { SD } \\
\text { Range }\end{array}$ & $\begin{array}{l}10: 3 \\
0: 5 \\
9: 4-11: 2\end{array}$ & $\begin{array}{l}38.5 \\
6.2 \\
27-46\end{array}$ & $\begin{array}{l}6.86 \\
0.36\end{array}$ & \\
\hline High load $(n=23)$ & $\begin{array}{l}\text { M } \\
\text { SD } \\
\text { Range }\end{array}$ & $\begin{array}{l}10: 2 \\
0: 3 \\
9: 5-10: 8\end{array}$ & $\begin{array}{l}38.2 \\
6.4 \\
27-49\end{array}$ & $\begin{array}{l}6.65 \\
0.57\end{array}$ & \\
\hline
\end{tabular}

Note:

$\mathrm{CA}=$ Chronological Age

SCQ = Social Communication Questionnaire

Stimuli and Procedure

Microsoft Visual Basic (version 6) was used to create computer-based stimuli that were presented on an IBM Lenovo Thinkpad 14.1" personal laptop (1440 x 900 pixel resolution). The participant's chair was positioned directly in front of the computer screen such that viewing distance was $60 \mathrm{~cm}$.

Target displays consisted of a black cross (RGB: $0,0,0)$, centered at fixation, and appearing on a white background (RGB: $255,255,255)$, with either the horizontal (H) or the vertical (V) line of the cross being longer than the other one (presentation was randomised across the first 6 trials, and counterbalanced across participants on trial 7). Perceptual load of the primary visual task was manipulated by increasing the complexity of perceptual operations involved in the line discrimination task (Lavie, 2005). This was achieved by adjusting the visual angle of one of the arms of the target cross, so that perceptual identification was more demanding on attention. In the high perceptual load condition, a cross with a shorter arm subtending $2.4^{\circ}$ and a longer arm subtending $3.9^{\circ}$ appeared, whereas in the low load condition, a cross with a shorter arm subtending $0.9^{\circ}$ and a longer arm subtending $3.9^{\circ}$ appeared (see Figure 1). This manipulation of perceptual load has previously been successfully applied in TD adults in an inattentional blindness (Cartwright-Finch and Lavie, 2007) and inattentional deafness paradigm (Macdonald and Lavie, 2011).

All children were tested individually in a room at their school. Prior to starting the computer task, the experimenter made sure that each child was able to correctly discriminate which line of a cross presented on an A4 sheet of paper was the longer one. Participants were then told that the cross would only appear very briefly on the screen, and that they need to fixate on the middle of screen and then tell the experimenter which line of the cross was longest. They were also told that they would need to wear headphones as part of the experiment. None of the children enquired further about the purpose of the headphones or whether they were going to hear something. 
Each of the six experimental trials began with the presentation of a fixation circle subtending $0.15^{\circ}$ in the middle of the screen $(1500 \mathrm{~ms})$; followed by a blank screen ( $\left.96 \mathrm{~ms}\right)$, a centrally located target cross (170ms), and finally a visual mask (496ms). A blank screen was then displayed while participants provided their verbal responses. This procedure was kept consistent across all eight trials (see Figure 2 below).

\section{Fig 1}

Illustration of stimuli used in (a) the low perceptual load condition and (b) high perceptual load condition

a)
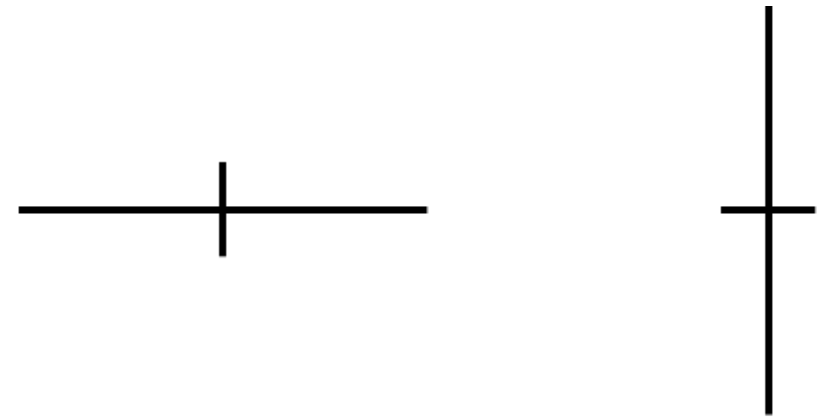

b)
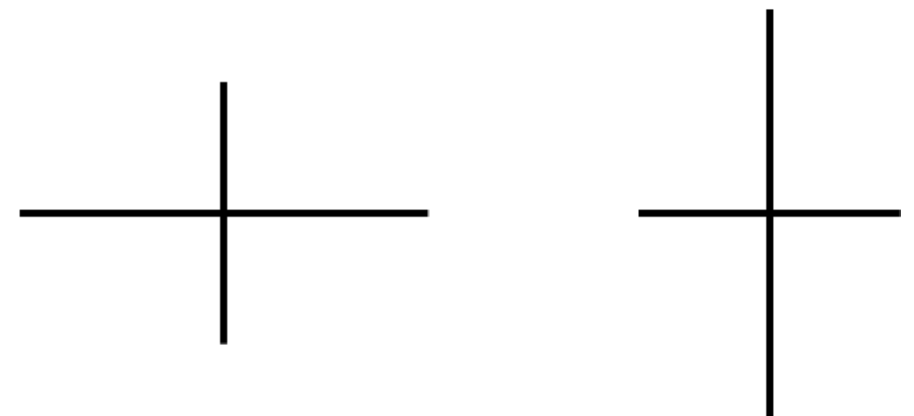

Note: In each perceptual load condition, presentation of the two crosses was randomised across trials 
Fig 2

Procedure of experimental trials

Trials 1-6

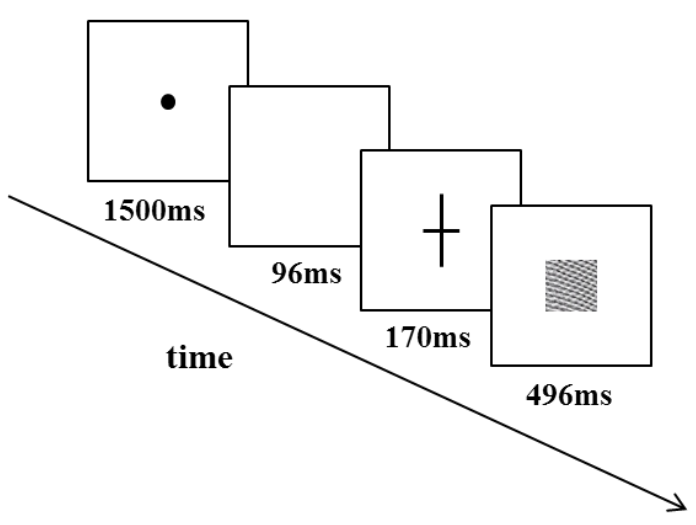

(1)

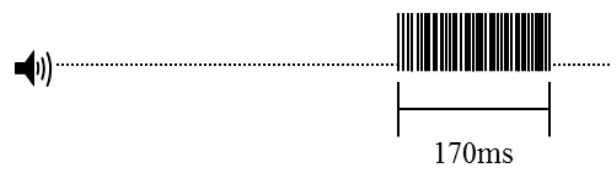

$\underline{\text { Trials } 7 \& 8 \text { (Critical \& control trial }}$

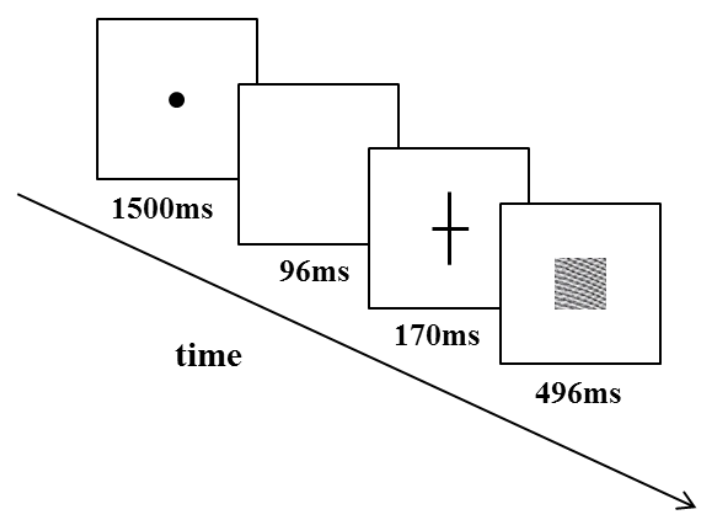

Responses to the cross task were either obtained by children's verbal responses referring to the lines as "the one going up and down" $(\mathrm{H})$ vs. "the one going across" $(\mathrm{V})$, pointing to visual aids placed next to the computer depicting each possible target cross scenario, or making appropriate hand gestures. The experimenter then entered their response on the computer. On the seventh and critical trial, the same procedure applied, but an auditory stimulus was played concurrently with presenting the cross. Responses to the cross task were recorded as in the previous trials, yet participants were also asked whether they had noticed anything else. This is the standard way of assessing awareness on the critical trial in inattentional blindness/deafness paradigms (e.g. Simons and Chabris, 1999; Memmert, 2006; Remington et al. 2014; Cartwright-Finch and Lavie, 2007; Macdonald and Lavie, 2011). Participants responded verbally, giving details of the critical stimulus (i.e. imitating the beep sound) where possible. The auditory stimulus used was a $180-\mathrm{Hz}$ pure tone of $69.4 \mathrm{~dB}$ played through a pair of Sennheiser HD 25-1-II stereo headphones. The intensity level was measured prior to the experiment by a Bruel \& Kjaer 4153 artificial ear together with an Ono Sokki CF-350Z spectrum analyser. The critical trial was subsequently repeated in a control trial, which measured awareness of the critical stimulus in absence of attention to the visual task. Participants were told prior to the control trial to ignore the cross stimulus and instead attend to any other stimulus they might notice. Only those participants who successfully identified the critical stimulus on the control trial were included in further analyses. Data from four children (2 TD and 2 ASD children) was excluded prior to analysis due to an inability to notice the critical stimulus on the control trial (no change in final sample size reported earlier).

\section{Results}

Cross task performance

A 2x2 between-subjects ANOVA was conducted to analyse the frequency of correct responses on the line discrimination task according to group (ASD vs. TD) and perceptual load condition (high load vs. low load). The results indicated no significant main effect of group (ASD: $M=6.62$; TD: $M=6.75$ ), $F(1,66)=1.201, \mathrm{p}=.277, \mathrm{n}_{\mathrm{p}}{ }^{2}=.02$; no main effect of condition (high load: $M=6.63$; low load: $M=6.77$ ), $F(1,66)=1.048, p=.310, \eta_{\mathrm{p}}^{2}=.02$; and no significant interaction between group and condition, $F(1,62)=0.317, \mathrm{p}=.575, \mathrm{n}_{\mathrm{p}}^{2}=.005$. 
Awareness of critical stimulus

Participants were considered to be aware of the critical stimulus (CS) if they reported hearing something on the critical trial, and were able to accurately describe the auditory stimulus verbally or by imitating the beep sound. Awareness rates as a function of perceptual load and group are shown in Figure 3.

\section{Fig 3}

Percentage of participants reporting awareness of the auditory stimulus (critical stimulus: CS) according to perceptual load and diagnostic group (error bars: standard error)

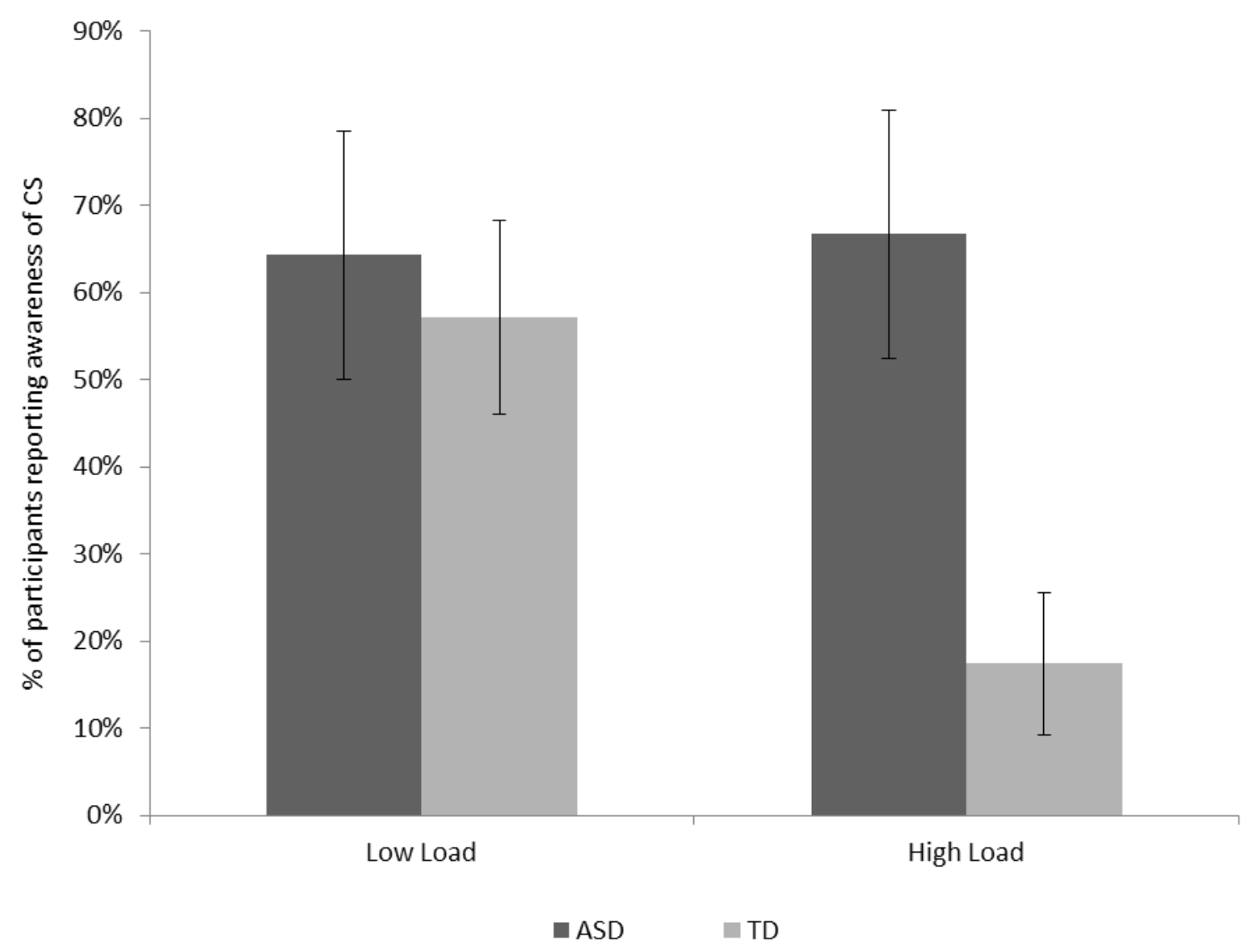

Detection

Due to the binary nature of the dependent variable, a hierarchical logistic regression analysis was carried out to predict awareness of the auditory stimulus ( $0=$ "No", $1=$ "Yes") according to perceptual load, diagnostic group, and their interaction. In a first step, an intercept-only model was compared against a model with perceptual load and diagnostic group as predictors. The latter model was subsequently compared to a full model including the interaction between perceptual load and diagnostic group. All statistical analyses used maximum likelihood ratio tests (model comparisons based on differences in -2 log likelihood) rather than the Wald statistic generally reported in hierarchical logistic regression analyses in order to account for a smaller sample size. The statistical cutoff criterion for all predictors was set at .05 .

The first regression model predicting awareness of the critical stimulus (CS: unexpected auditory stimulus) from perceptual load and diagnostic group performed significantly better overall than the intercept-only model, $\chi^{2}(2)=10.054, p=.007$. The Hosmer and Lemeshow test also indicated a good fit of the model to the data, $\chi^{2}(2)=3.322, p=.190$. When controlling for the effect of group, there was a significant effect of perceptual load on 
awareness, $\chi^{2}(1)=4.467, p=.035$, with participants in the high perceptual load condition being 0.342 times less likely to notice the stimulus than participants in the low perceptual load condition.

Table 2 Logistic regression analysis predicting awareness of the critical stimulus from perceptual load condition and diagnostic group

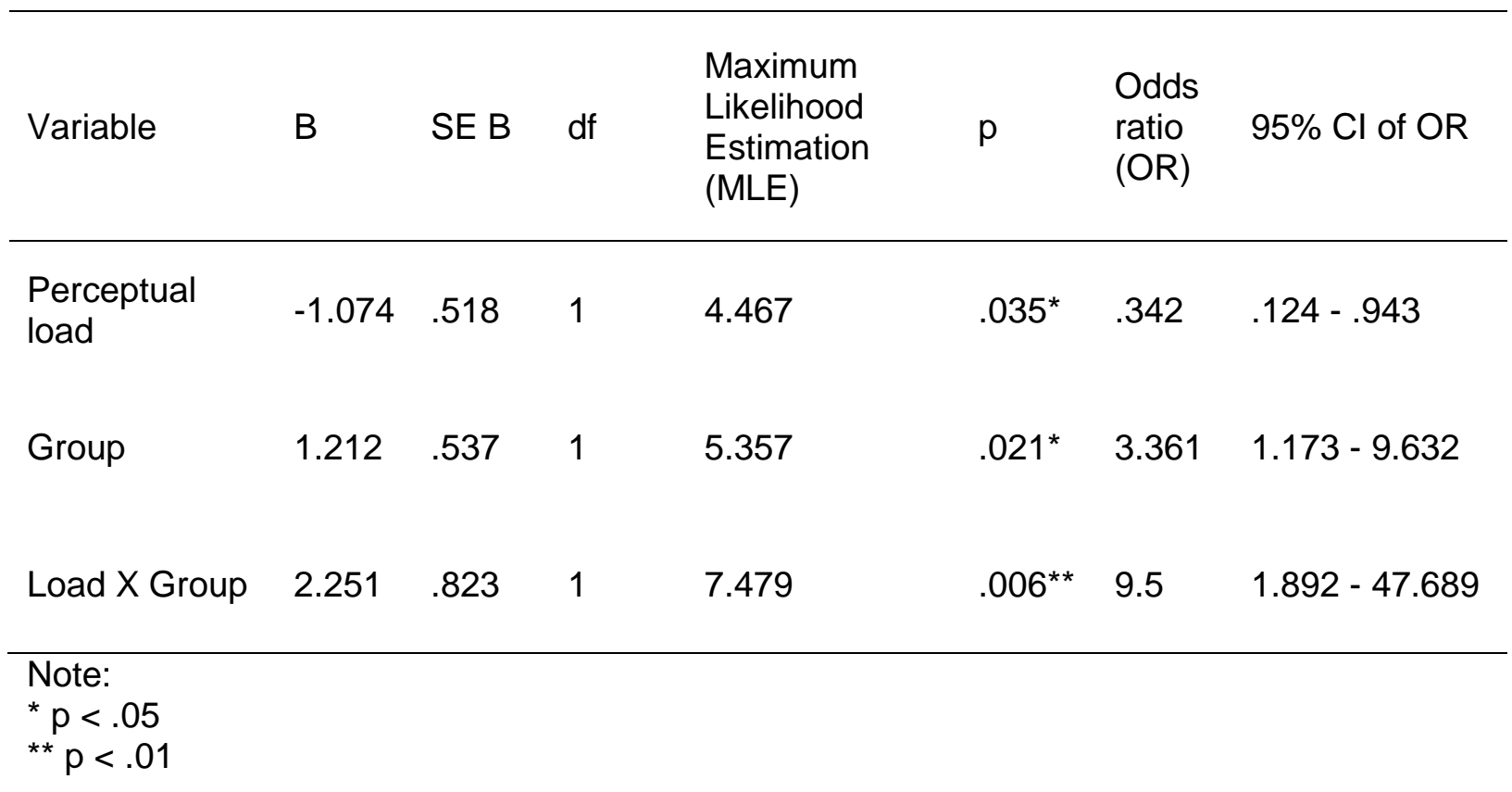

Diagnostic group also significantly predicted awareness of the CS, $\chi^{2}(1)=5.357, p=.021$ with participants in the ASD group being 3.4 times more likely to notice the critical stimulus (for maximum likelihood ratio tests, odds ratio and $\mathrm{Cl}$ of odds ratio see Table 2).

The interaction between perceptual load and diagnostic group was tested by comparing a full model that included all predictors (perceptual load, group, and their interaction), against a reduced model with only load and group as predictors. The likelihood ratio test revealed a significant interaction between perceptual load and group, $\chi^{2}(1)=7.479, p=.006$, suggesting a different effect of perceptual load on detection between groups. In particular, whereas in the low perceptual load condition detection rates did not differ between groups (TD: $57 \%$ vs. ASD: $64 \%$ ), $\chi^{2}(1)=.179, p=.672$, detection rates were significantly reduced for the TD group compared to the ASD group in the high perceptual load condition (TD: $17 \%$ vs. ASD: $66 \%), \chi^{2}(1)=8.498, p=.004$. An additional logistic regression analysis also confirmed that neither non-verbal ability scores nor scores on the SCQ was a significant predictor (both $p>.05$ ) of awareness rates of the CS in the ASD group.

\section{Discussion}

This study demonstrates greater awareness for an unexpected auditory stimulus (critical stimulus: CS) overall and a reduced effect of visual perceptual load on CS awareness in children with ASD compared to typically developing (TD) children. Importantly, the increased rates of CS awareness in children with ASD, when visual perceptual load was high, are not likely to be explained by a trade-off in line discrimination accuracy. These findings suggest an increased perceptual capacity in children with ASD that operates across sensory modalities.

According to Load theory, awareness of task-irrelevant stimuli depends on whether sufficient perceptual capacity is available to process additional information, after processing of task relevant stimuli has finished. Thus, a high perceptual load task should result in low awareness rates for task-irrelevant stimuli, as all processing capacity is already exhausted 
by performing a primary task. In contrast, a low perceptual load task should result in high rates of awareness of task-irrelevant stimuli as spare capacity automatically "spills over" to process additional task-irrelevant stimuli (Lavie, 2005). As awareness rates for the unexpected auditory stimulus (CS) remained unaffected by increasing perceptual load of the central task in children with ASD, yet dropped significantly in TD children, the findings suggest that children with ASD had processing resources left-over to also attend to the CS, whereas TD children did not. Our findings therefore provide further support for the hypothesis that individuals with ASD have a higher perceptual capacity.

Both groups showed equally high performance rates in the high as well as the low perceptual load conditions of the line discrimination task. Of course this was not surprising given that we excluded any participant who did not meet our criteria of 5 out of 6 correct discriminations on non-critical trials and a correct discrimination on the critical trial (when the CS was presented). It is worth noting though that only three participants failed to reach these inclusion criteria. All three were children with ASD in the high perceptual load condition. It is not possible to tell exactly why these three participants did not perform well on the line discrimination task, this may have been due to a lack of motivation, a misunderstanding of instructions or a genuine difficulty in making discriminations. However, for the purpose of this study it was important only to include participants who we could be confident were successfully performing the central task when the CS appeared. Any differences in CS awareness are therefore unlikely to be due to a lack of engagement with the central task. The participants' performance on the central task was only measured in terms of accuracy on the line discrimination task and not in terms of reaction time. In the current study we decided that it was important to keep the number of trials to a minimum in order to ensure that as many children as possible continued to engage with the task. Given the small number of trials and therefore larger variability in reaction times across trials, it is unlikely that in the current task design, reaction time would have been a reliable measure of task performance. Future research could attempt to increase the number of trials and also measure participants' reaction times in order to obtain a more nuanced measure of task performance. Such reaction time data could help rule out the possibility that increased detection rates occurred because the ASD participants had more time to divert attentional resources from the primary task to the unexpected auditory stimulus. Whilst we cannot completely dismiss this explanation with the current data, it seems unlikely given the experimental set-up. The auditory stimulus was a single, unexpected event and as a result, participants were neither expecting the additional auditory stimulus nor the surprise question following the critical trial. As the auditory stimulus was unexpected, it seems unlikely that task strategies influenced detection rates such that participants prepared to divert attention to the unattended modality prior to the critical trial. The results also indicated that overall cognitive abilities (non-verbal ability scores) and ASD symptomatology (SCQ scores) were not able to account for the increased awareness rates of the CS in the ASD group, suggesting that the results were not simply driven by a subgroup of more able children with ASD (i.e. those with higher non-verbal ability scores and lower SCQ scores). However, we do note that our sample, as well as those from previous studies (Remington et al. 2009, 2012b; Swettenham et al. 2014) only included participants with ASD that were matched on non-verbal ability scores with typically developing participants. To date there have been no studies examining perceptual capacity in children with ASD who also have learning difficulties. Could the difference in awareness rates between the ASD and TD group be due to under reporting by the TD group? For example, there is evidence that individuals with autism are less concerned about social pressures and more ready to report veridical experience (e.g., Bowler \& Worley, 1994). This seems unlikely as a main explanation for the findings given that the difference between the groups in reporting rates occurs in one load condition (high load) but not in another (low load) despite the auditory stimulus being the same in both. 
Reduced awareness rates in TD children on the other hand cannot be attributed to the low intensity of the auditory stimulus. On both the critical and control trial, the auditory stimulus was played at hearing levels well above threshold and was therefore easily perceivable, and all participants noticed the auditory stimulus and were able to describe it on the control trial when their attention was not focused on the primary visual task. The observed group effect is also unlikely to be caused by any differences in cognitive abilities as all participants were carefully matched for performance on the Raven's Progressive Matrices.

The pattern of results for the typically developing children is in line with load theory and previous demonstrations of the effect of visual perceptual load on inattentional deafness in typically developing adults (Macdonald and Lavie, 2011). Our study therefore demonstrates the cross modal effect of perceptual load on selective attention in typically developing children. That is, the extent to which children were able to notice the presence of an unexpected auditory stimulus was dependent on whether sufficient attentional resources were available after the processing of a primary visual task. This parallels findings within the visual modality on an inattentional blindness task, where typically developing children also showed load-dependent effects on awareness of a visual CS (Remington et al. 2014). Furthermore, on a visual search task with varying levels of perceptual load, both children and adults exhibited interference effects from distracting flankers under low, but not high levels of visual perceptual load (Huang-Pollock et al. 2002).

The finding of reduced inattentional deafness under load in children with ASD extends existing work on the effect of perceptual load on selective attention in ASD. In particular, whereas previous demonstrations of an increased perceptual capacity in ASD have only been within the visual domain (Remington et al. 2009; 2012b; Swettenham et al. 2014), the current findings suggest that this increased capacity operates across sensory modalities. However, the one-trial nature of the inattentional deafness paradigm means that one should be cautious to not overstate the conclusions of this single study. Whilst our findings suggest a perceptual advantage for children with ASD attending to information in two modalities, there is some evidence in the literature that suggests that perceptual processing may be impaired in ASD when participants are required to integrate stimuli from different sensory modalities (see Marco et al. 2011, for a review). Whereas typically developing individuals generally benefit from the presentation of multisensory stimuli, showing enhanced performance on detection and discrimination tasks (Perrott, Saberi, Brown and Strybel, 1990; Spence and Driver, 1997; Stein, London, Wilkinson and Price, 1996), individuals with ASD often show reduced behavioural facilitation and reduced cortical activation in response to multisensory stimuli (Russo et al. 2010; Brandwein et al. 2013). Brandwein et al. (2013) for example, found that children with ASD were slower to respond to audio-visual (AV) stimulus pairs on a simple reaction time task than TD children, and showed less widespread cortical activation in the AV condition, particularly at early stages of information processing. Integration of multisensory stimuli in the context of speech perception also seems to be impaired in ASD. For example, individuals with ASD are reported to be less susceptible to the McGurk effect (De Gelder et al. 1991; Smith and Bennetto, 2007, but also see larocci et al. 2010; Williams et al. 2004), in which the simultaneous presentation of a speech sound (e.g. /ba/) with an incongruent visual stimulus (e.g. /va/) can result in inaccurate auditory perceptions. These findings indicate that individuals with ASD do not integrate visual and auditory information in the same manner as TD children. Others however have suggested that these differences are due to poorer lip reading abilities in ASD (larocci et al. 2010; Williams et al. 2004), as well as the confounding effects of using social (e.g. faces) and/or linguistic stimuli, which are inherently more difficult for individuals with ASD to process. Indeed, when using basic, non-social stimuli, Van der Smagt et al. (2007) found that children with ASD were as susceptible as TD children to the sound-induced double-flash illusion where the number of beeps has been shown to produce the percept of additional illusory flashes (Shams et al. 2002). 
However, the present study and the above work on multisensory integration (MSI) in ASD differ considerably in terms of task requirements. While both the MSI tasks and our current task involve the processing of simultaneously presented stimuli from different sensory modalities, a typical MSI task is a perceptual task where perception of a stimulus in one modality is enhanced by the presence of a stimulus in another modality through a process of integration. The inattentional deafness procedure employed here is a selective attention task where the additional auditory stimulus does not facilitate the processing of the visual stimulus (or vice versa). Instead, the emphasis is on whether an unexpected and therefore genuinely unattended auditory stimulus captures attention according to the level of visual perceptual load of a central task. Also, whereas in MSI tasks stimuli from both modalities are generally presented throughout the experiment (and hence receive deliberate attention), the complete absence of expectation of the additional stimulus in our design means that processing of the additional stimulus is not dependent on any deliberate attentional allocation towards it.

The current study demonstrated that visual perceptual load had a different effect on awareness of a task-irrelevant auditory stimulus in children with ASD compared to TD children. In future studies, it would be interesting to examine whether a stimulus that conveys biological and socially relevant information (e.g. a person greeting another person) would produce similar results. In the visual domain, there is evidence that social stimuli (e.g. faces) do not capture attention to the same extent in ASD compared to TD children. Remington, Campbell and Swettenham (2012a) for instance demonstrated that unlike TD adults who processed distracting faces regardless of the perceptual load of a task, adults with ASD only processed distracting faces at low levels of perceptual load, but not at high levels of perceptual load. These findings suggest that in the typical population, social stimuli have a "special status" and are processed in an automatic fashion regardless of the perceptual load of a relevant task (see also Lavie et al. 2003). From these results, it could be predicted that under high perceptual load, a socially relevant but unexpected auditory stimulus captures attention in TD individuals, yet in ASD individuals, receives less processing priority and therefore leads to lower awareness rates.

Finally, our findings might also provide an explanation for the common observation by parents and clinicians that children with ASD experience difficulties and even distress if the environment features too many sensory stimuli. An increased perceptual capacity in ASD that operates across sensory modalities would allow children with ASD to process more sensory information at any one time, even if in some circumstances this information is distracting for their current task behaviour. Educational and therapeutic programmes should therefore aim to exploit the advantages of such an enhancement, but equally provide a learning environment within which the potentially detrimental effects are minimised.

\section{Acknowledgments:}

This research was supported by an Economic and Social Research Council studentship. We thank all pupils and staff members for their efforts to participate in the study.

\section{References}

Adams, N. C., \& Jarrold, C. (2012). Inhibition in autism: children with autism have difficulty inhibiting irrelevant distractors but not prepotent responses. Journal of Autism and Developmental Disorders, 42, 1052-1063. doi: 10.1007/s10803-011-1345-3

Association, A. P. (1994). Diagnostic and Statistical Manual of Mental Disorders (4th ed.). Washington DC: American Psychiatric Association.

Association, A. P. (2013). Diagnostic and statistical manual of mental disorders (5th ed.). Washington, DC: Author.

Bonnel, A., McAdams, S., Smith, B., Berthiaume, C., Bertone, A., Ciocca, V., . . Mottron, L. (2010). Enhanced pure-tone pitch discrimination among persons with autism but not 
Asperger syndrome. Neuropsychologia, 48(9), 2465-2475. doi: 10.1016/j.neuropsychologia.2010.04.020

Bowler, D. M., \& Worley, K. (1994). Susceptibility to social influence in adults with Asperger's syndrome: A research note. Journal of Child Psychology and Psychiatry, 35(4), 689697. doi: 10.1111/j.1469-7610.1994.tb01214.x

Brandwein, A. B., Foxe, J. J., Butler, J. S., Russo, N. N., Altschuler, T. S., Gomes, H., \& Molholm, S. (2013). The development of multisensory integration in high-functioning autism: high-density electrical mapping and psychophysical measures reveal impairments in the processing of audiovisual inputs. Cerebral Cortex, 23, 1329-1341. doi: $10.1093 /$ cercor/bhs 109

Burack, J. A. (1994). Selective attention deficits in persons with autism: preliminary evidence of an inefficient attentional lens. Journal of Abnormal Psychology, 103(3), 535-543. doi: 10.1037/0021-843X.103.3.535

Carmel, D., Thorne, J. D., Rees, G., \& Lavie, N. (2011). Perceptual load alters visual excitability. Journal of Experimental Psychology: Human Perception and Performance, 37(5), 1350. doi: 10.1037/a0024320

Cartwright-Finch, U., \& Lavie, N. (2007). The role of perceptual load in inattentional blindness. Cognition, 102, 321-340. doi: 10.1016/j.cognition.2006.01.002

Casey, B., Gordon, C., Mannheim, G. B., \& Rumsey, J. M. (1993). Dysfunctional attention in autistic savants. Journal of Clinical and Experimental Neuropsychology, 15(6), 933946. doi: 10.1080/01688639308402609

Christ, S. E., Holt, D. D., White, D. A., \& Green, L. (2007). Inhibitory control in children with autism spectrum disorder. Journal of Autism and Developmental Disorders, 37, 11551165. doi: 10.1007/s10803-006-0259-y

Ciesielski, K. T., Knight, J. E., Prince, R. J., Harris, R. J., \& Handmaker, S. D. (1995). Eventrelated potentials in cross-modal divided attention in autism. Neuropsychologia, 33(2), 225-246. doi: 10.1016/0028-3932(94)00094-6

De Gelder, B. d., Vroomen, J., \& Van der Heide, L. (1991). Face recognition and lip-reading in autism. European Journal of Cognitive Psychology, 3(1), 69-86. doi: $10.1080 / 09541449108406220$

Eriksen, B. A., \& Eriksen, C. W. (1974). Effects of noise letters upon the identification of a target letter in a nonsearch task. Perception \& Psychophysics, 16(1), 143-149. doi: 10.3758/BF03203267

Frith, U. (1989). Autism: Explaining the enigma. Oxford: Blackwell.

Geurts, H. M., Luman, M., \& Van Meel, C. S. (2008). What's in a game: the effect of social motivation on interference control in boys with ADHD and autism spectrum disorders. Journal of Child Psychology and Psychiatry, 49(8), 848-857. doi: 10.1111/j.14697610.2008.01916.x

Handy, T. C., Soltani, M., \& Mangun, G. R. (2001). Perceptual load and visuocortical processing: Event-related potentials reveal sensory-level selection. Psychological Science, 12(3), 213-218. doi: 10.1111/1467-9280.00338

Happé, F., \& Frith, U. (2006). The weak coherence account: detail-focused cognitive style in autism spectrum disorders. Journal of Autism and Developmental Disorders, 36(1), 5-25. doi: 10.1007/s10803-005-0039-0

Happé, F. G. (1996). Studying weak central coherence at low levels: children with autism do not succumb to visual illusions. A research note. Journal of Child Psychology and Psychiatry, 37(7), 873-877. doi: 10.1111/j.1469-7610.1996.tb01483.x

Heaton, P. (2003). Pitch memory, labelling and disembedding in autism. Journal of Child Psychology and Psychiatry, 44(4), 543-551. doi: 10.1111/1469-7610.00143

Huang-Pollock, C. L., Carr, T. H., \& Nigg, J. T. (2002). Development of selective attention: Perceptual load influences early versus late attentional selection in children and adults. Developmental Psychology, 38, 363-375. doi: 10.1037//0012-1649.38.3.363

larocci, G., Rombough, A., Yager, J., Weeks, D. J., \& Chua, R. (2010). Visual influences on speech perception in children with autism. Autism, 14, 305-320. doi: $10.1177 / 1362361309353615$ 
Johnson, M. H. (2005). Developmental Cognitive Neuroscience (2nd ed.). Oxford: Blackwell.

Jones, C. R., Happé, F., Baird, G., Simonoff, E., Marsden, A. J., Tregay, J., . . Charman, T. (2009). Auditory discrimination and auditory sensory behaviours in autism spectrum disorders. Neuropsychologia, 47(13), 2850-2858. doi: 10.1016/j.neuropsychologia.2009.06.015

Lavie, N. (1995). Perceptual load as a necessary condition for selective attention. Journal of Experimental Psychology. Human Perception and Performance, 21, 451-468. doi: 10.1037/0096-1523.21.3.451

Lavie, N. (2005). Distracted and confused?: Selective attention under load. Trends in Cognitive Sciences, 9, 75-82. doi: 10.1016/j.tics.2004.12.004

Lavie, N. (2010). Attention, distraction, and cognitive control under load. Current Directions in Psychological Science, 19(3), 143-148. doi: 10.1177/0963721410370295

Lavie, N., \& de Fockert, J. W. (2003). Contrasting effects of sensory limits and capacity limits in visual selective attention. Perception \& Psychophysics, 65, 202-212. doi: 10.3758/BF03194795

Lavie, N., Ro, T., \& Russell, C. (2003). The Role of Perceptual Load in Processing Distractor Faces. Psychological Science, 14, 510-515. doi: 10.1111/1467-9280.03453

Lavie, N., \& Torralbo, A. (2010). Dilution: atheoretical burden or just load? A reply to Tsal and Benoni (2010). Journal of Experimental Psychology. Human Perception and Performance, 36, 1657-1664; discussion 1665-1658. doi: 10.1037/a0020733

Lavie, N., \& Tsal, Y. (1994). Perceptual load as a major determinant of the locus of selection in visual attention. Perception \& Psychophysics, 56, 183-197. doi: 10.3758/BF03213897

Macdonald, J. S. P., \& Lavie, N. (2011). Visual perceptual load induces inattentional deafness. Attention, Perception \& Psychophysics, 73, 1780-1789. doi: 10.3758/s13414-011-0144-4

Marco, E. J., Hinkley, L. B. N., Hill, S. S., \& Nagarajan, S. S. (2011). Sensory Processing in Autism: A Review of Neurophysiologic Findings. Pediatric Research, 69, 48R-54R. doi: 10.1203/PDR.0b013e3182130c54

Matusz, P. J., Broadbent, H., Ferrari, J., Forrest, B., Merkley, R., \& Scerif, G. (2015). Multimodal distraction: Insights from children's limited attention. Cognition, 136, 156-165. doi: 10.1016/j.cognition.2014.11.031

Memmert, D. (2006). The effects of eye movements, age, and expertise on inattentional blindness. Consciousness and Cognition, 15, 620-627. doi: 10.1016/j.concog.2006.01.001

Mottron, L., Dawson, M., Soulieres, I., Hubert, B., \& Burack, J. (2006). Enhanced perceptual functioning in autism: an update, and eight principles of autistic perception. Journal of Autism and Developmental Disorders, 36(1), 27-43. doi: 10.1007/s10803-005-0040-7

Murray, J. E., \& Jones, C. (2002). Attention to local form information can prevent access to semantic information. The Quarterly Journal of Experimental Psychology Section A: Human Experimental Psychology, 55(2), 609-625. doi: 10.1080/02724980143000370

O'Riordan, M. (2004). Superior visual search in adults with autism. Autism, 8, 229-248. doi: $10.1177 / 1362361304045219$

O'Riordan, M., Plaisted, K., Driver, J., \& Baron-Cohen, S. (2001). Superior Visual Search in Autism. Journal of Experimental Psychology: Human Perception and Performance, 27, 719-730. doi: 10.1037/0096-1523.27.3.719

O'Connor, K. (2012). Auditory processing in autism spectrum disorder: a review. Neuroscience \& Biobehavioral Reviews, 36(2), 836-854. doi: 10.1016/j.neubiorev.2011.11.008

Perrott, D. R., Saberi, K., Brown, K., \& Strybel, T. Z. (1990). Auditory psychomotor coordination and visual search performance. Perception \& Psychophysics, 48(3), 214-226. doi: 10.3758/BF03211521

Plaisted, K., O'Riordan, M., \& Baron-Cohen, S. (1998). Enhanced visual search for a conjunctive target in autism: A research note. Journal of Child Psychology and Psychiatry, 39(5), 777-783. doi: 10.1017/ S0021963098002613 
Plaisted, K., Swettenham, J., \& Rees, L. (1999). Children with autism show local precedence in a divided attention task and global precedence in a selective attention task. Journal of Child Psychology and Psychiatry, 40, 733-742. doi: doi:10.1111/14697610.00489

Raveh, D., \& Lavie, N. (2015). Load-induced inattentional deafness. Attention, Perception, \& Psychophysics, 77(2), 483-492. doi: 10.3758/s13414-014-0776-2

Raven, J., Raven, J. C., \& Court, J. H. (1998). Manual for Raven's progressive matrices and vocabulary scales - Section 1: General overview. Oxford, United Kingdom: Oxford Psychologists Press.

Remington, A., Campbell, R., \& Swettenham, J. (2012a). Attentional status of faces for people with autism spectrum disorder. Autism, 16, 59-73. doi: $10.1177 / 1362361311409257$

Remington, A., Cartwright-Finch, U., \& Lavie, N. (2014). I can see clearly now: the effects of age and perceptual load on inattentional blindness. Frontiers in Human Neuroscience, 8, 229. doi: 10.3389/fnhum.2014.00229

Remington, A., Swettenham, J., Campbell, R., \& Coleman, M. (2009). Selective attention and perceptual load in autism spectrum disorder. Psychological Science, 20, 13881393. doi: 10.1111/j.1467-9280.2009.02454.x

Remington, A. M., Swettenham, J. G., \& Lavie, N. (2012b). Lightening the load: Perceptual load impairs visual detection in typical adults but not in autism. Journal of Abnormal Psychology, 121, 544-551. doi: 10.1037/a0027670

Russo, N., Foxe, J. J., Brandwein, A. B., Altschuler, T., Gomes, H., \& Molholm, S. (2010). Multisensory processing in children with autism: high-density electrical mapping of auditory-somatosensory integration. Autism Research, 3, 253-267. doi: 10.1002/aur.152

Rutter, M., Bailey, A., \& Lord, C. (2003). Social Communication Questionnaire. Los Angeles, CA: Western Psychological Services.

Schwartz, S., Vuilleumier, P., Hutton, C., Maravita, A., Dolan, R. J., \& Driver, J. (2005). Attentional load and sensory competition in human vision: modulation of fMRI responses by load at fixation during task-irrelevant stimulation in the peripheral visual field. Cerebral Cortex, 15(6), 770-786. doi: 10.1093/cercor/bhh178

Shah, A., \& Frith, U. (1993). Why do autistic individuals show superior performance on the block design task? Journal of Child Psychology and Psychiatry, 32, 1351-1364. doi: 10.1111/j.1469-7610.1993.tb02095.x

Shams, L., Kamitani, Y., \& Shimojo, S. (2002). Visual illusion induced by sound. Cognitive Brain Research, 14(1), 147-152. doi: 10.1016/S0926-6410(02)00069-1

Simmons, D. R., Robertson, A. E., McKay, L. S., Toal, E., McAleer, P., \& Pollick, F. E. (2009). Vision in autism spectrum disorders. Vision research, 49(22), 2705-2739. doi: 10.1016/j.visres.2009.08.005

Simons, D. J., \& Chabris, C. F. (1999). Gorillas in our midst: sustained inattentional blindness for dynamic events. Perception, 28, 1059-1074. doi: 10.1068/p2952

Smith, E. G., \& Bennetto, L. (2007). Audiovisual speech integration and lipreading in autism. Journal of Child Psychology and Psychiatry, 48(8), 813-821. doi: 10.1111/j.14697610.2007.01766.x

Spence, C., \& Driver, J. (1997). On measuring selective attention to an expected sensory modality. Perception \& Psychophysics, 59, 389-403. doi: 10.3758/BF03211906

Stein, B., London, N., Wilkinson, L., \& Price, D. (1996). Enhancement of perceived visual intensity by auditory stimuli: a psychophysical analysis. Journal of Cognitive Neuroscience, 8(6), 497-506. doi: 10.1162/jocn.1996.8.6.497

Swettenham, J., Remington, A., Murphy, P., Feuerstein, M., Grim, K., \& Lavie, N. (2014). Seeing the Unseen: Autism Involves Reduced Susceptibility to Inattentional Blindness. Neuropsychology. doi: 10.1037/neu0000042

Theeuwes, J., Kramer, A. F., \& Belopolsky, A. V. (2004). Attentional set interacts with perceptual load in visual search. Psychonomic Bulletin \& Review, 11(4), 697-702. doi: 10.3758/BF03196622 
Treisman, A. M., \& Gelade, G. (1980). A feature-integration theory of attention. Cognitive Psychology, 12(1), 97-136. doi: 10.1016/0010-0285(80)90005-5

Tsal, Y., \& Benoni, H. (2010a). Diluting the burden of load: perceptual load effects are simply dilution effects. Journal of Experimental Psychology. Human Perception and Performance, 36, 1645-1656. doi: 10.1037/a0018172

Tsal, Y., \& Benoni, H. (2010b). Much dilution little load in Lavie and Torralbo's (2010) response: A reply. Journal of Experimental Psychology: Human Perception and Performance, 36, 1665-1668. doi: 10.1037/a0021907

van der Smagt, M. J., van Engeland, H., \& Kemner, C. (2007). Brief report: can you see what is not there? low-level auditory-visual integration in autism spectrum disorder. Journal of Autism and Developmental Disorders, 37, 2014-2019. doi: 10.1007/s10803-0060346-0

Williams, J. H., Massaro, D. W., Peel, N. J., Bosseler, A., \& Suddendorf, T. (2004). Visualauditory integration during speech imitation in autism. Research in Developmental Disabilities, 25(6), 559-575. doi: 10.1016/j.ridd.2004.01.008 